\title{
El vínculo relacional de pareja y las redes sociales: una mirada desde la cotidianidad
}

\section{The relational bond and social networks: a gaze from the everydayness}

\author{
Gabriel Balaguera-Rojas \\ $\triangle$ gabriel.balaguera@usantotomas.edu.co \\ Universidad Santo Tomás, Colombia. \\ Nolly Paola Forero-Trujillo (iD \\ $\triangle$ nolly.forero@usantotomas.edu.co \\ Universidad Santo Tomás, Colombia. \\ Vanessa Buitrago-Márquez (iD) \\ $\triangle$ vanessa.buitrago@usantotomas.edu.co \\ Universidad Santo Tomás, Colombia.
}

Lizeth Dayanna Cruz-Domínguez

$\triangle$ Lizeth.cruz@usantotomas.edu.co

Recibido: $18-07-2018$

Universidad Santo Tomás, Colombia.

Aceptado: 11-12-2018

Publicado: 17-12-2018

\section{Resumen}

Objetivo: identificar las comprensiones entre el uso de las redes sociales y el vínculo de las parejas desde la interacción cotidiana de los participantes en plataformas digitales. Método: la investigación se fundamenta en una metodología cualitativa que da paso a la interpretación de las significaciones de los usuarios, para así obtener diferentes posturas frente a la percepción que se tienen acerca de las redes sociales y la forma como estas afectan aspectos de la dinámica relacional. Resultados: se lograron identificar categorías emergentes a través de un enfoque sistémico del que se desprenden subcategorías, tales como la reciprocidad, la comunicación, la estabilidad y las fuentes de tensión; información que fue analizada a través de la interpretación de los datos desde las experiencias subjetivas de los intervinentes. Conclusiones: existe presencia de cambios en el vínculo relacional de la pareja desde el inicio de sus relaciones; las simbologías dentro de redes sociales como Facebook, Whatsapp e Instagram son utilizadas como representaciones de las emociones humanas y la codificación de la información se da a través de tipos de comunicación que estas ofrecen, como es el caso de la comunicación sincrónica y la comunicación asincrónica, con las cuales se fortalece o quebranta el vínculo relacional del sujeto.

Palabras clave: Relaciones de pareja, redes sociales, vínculos, comunicación, cotidianidad. 


\section{Abstract}

Objective: to identify the comprehensions between the use of social networks and the link of couples from the daily interaction of participants in digital platforms. Method: the research is based on a qualitative methodology that gives way to the interpretation of the meanings of the users, in order to obtain different positions regarding the perception they have about social networks and the way they affect aspects of relational dynamics. Results: emerging categories were identified through a systemic approach from which subcategories are derived, such as reciprocity, communication, stability and sources of tension; information was analyzed through the interpretation of data from the subjective experiences of the participants. Conclusions: there are changes in the relational bond of the couple since the beginning of their relationships; symbologies within social networks such as Facebook, Whatsapp and Instagram are used as representations of human emotions and the codification of information is given through types of communication that these offer, such as synchronous communication and asynchronous communication, with which the relational bond of the subject is strengthened or broken.

Keywords: Couple relationships, social media, link, communication, everydayness.

\section{Introducción}

La sociedad se ha desarrollado a tal punto de descubrir y enfrentar nuevas necesidades reflejadas en las redes sociales, para saciar carencias comunicativas y de distancia como una forma de adaptación humana. Esta sociedad tiene transformaciones desde dos dimensiones la Modernidad y las tecnologías en un medio de globalización. Es importante aclarar que al hablar de cambios no se trata de la conformación de nuevas sociedades o sociedades de red, de información o sociedades de riesgo, sino de nuevos aspectos que surgen en las sociedades que tienen distintas formas de relacionarse y que surgen como nuevas comprensiones de la interacción que crea las herramientas de las redes sociales en la comunicación que puede proporcionar el fortalecimiento o en el quebrantamiento del sistema relacional, según lo menciona Gainza (2003).

Teniendo en cuenta lo anterior, es preciso destacar que Colombia es uno de los países con menores restricciones de navegación para los usuarios; se ubica en el octavo puesto del ranking mundial de clasificación de las redes más libres. Los colombianos acceden a las redes sociales más utilizadas por lo menos diez veces al día, entre las cuales se destacan Facebook, con un 70,1\%; WhatsApp con 60,1\%, red que supera un millón de usuarios activos al mes; Instagram, con una población de 31,5\%; otras como YouTube, con un 51,6\%; Twitter en un 29,3\%, y MySpace con una participación del $6,2 \%$, según estadísticas que brinda el Ministerio de Tecnologías de la Información y las Comunicaciones (2015).

Ante estas tasas, se logra inferir que la sociedad colombiana se ve enmarcada en el diario vivir de sus ciudadanos por las redes sociales, por lo que se fundamenta la necesidad de abordar este fenómeno dentro de la dinámica relacional de pareja, ya que las redes sociales proporcionan un medio de interacción y expresión no solo personal, sino de la propia relación; es decir que se construye la relación a partir de su influencia, de acuerdo con Cuervo (2013). 
Cabe resaltar que la tecnología ha hecho de la vida cotidiana de las personas un común denominador respecto al tema de las redes sociales, por lo que las relaciones interperonales han tenido un cambio sustancial fundamentado en la comunicación, ya que el acceso a Internet responde a una elevada participación en el mundo, debido a la facilidad de conectividad entre las personas, según lo menciona Vallejos (s.f.). Es así que las redes forman lazos vinculantes en las relaciones de los usuarios que se crean a partir de la confianza entre ellos, lo que genera un lazo afectivo, según Urizar (2012). Para que este sea efectivo, debe estar mediado por un vínculo con tendencia a la homeostasis; es decir, que se llegue al equilibrio de adaptación a los cambios y a las cosas que tengan en común, lo cual redunda en el crecimiento y personificación adecuada de los roles en un subsistema, de acuerdo a lo mencionado por Castellanos (2015).

En este tipo de ambientes comunicativos, se generan situaciones que redundan en aspectos que son vistos como fuera de los cauces normales dentro de las relaciones de pareja, y se prestan para que se produzcan situaciones como las infidelidades, al ser las redes una ventana abierta a diferentes posibilidades de acercamientos relacionales que pueden llegar a materializare en una relación casual, de manera que los vínculos se fortalecen por la comunicación cercana que se presentan entre dos personas, lo cual llega a afectar la relación estable de pareja que tiene uno u otro usuario; así lo mencionan Casero y Algaba (2016).

Debido a lo anteriormente planteado, se acota que las redes sociales aportan una oportunidad al suplir la necesidad de interactuar y de mostrar una imagen de sí en la que la persona se expresa y se integra con iguales dándose a conocer mediante pequeñas características en cada perfíl que crean los usuarios. Esto convierte el uso de las redes sociales hoy en día en un fenómeno de masas, según lo afirman López, López y Galán (s.f).

Atendiendo a lo mencionado, se puede decir que el individuo está ligado directamente a un fenómeno en el que a través de la comunicación se expresan significados que atribuyen a la percepción del contexto, por lo que el conocimiento emerge de una relación directa en la que se intenta comprender e interpretar la realidad que se está investigando -como lo mencionan por Cornejo y Tapia (2011) - y se toman en cuenta elementos que pueden intervenir, transformar o aportar a dicha realidad, como las creencias, las reflexiones que se generan en las acciones. Los valores que surgen por la conciencia, cuando se habla de la esencia de la misma, generan reflexión de la realidad y se habla entonces a partir de una interacción constante expresa (Ricoy, 2006).

Finalmente, se tiene que los cambios que componen el sistema, comprendidos desde un fenómeno cambiante, se ajustan, modifican y reorganizan en torno al sistema relacional en el cual el vínculo puede llegar a ser favorecido desde los cambios que emergen de este, hasta el punto de llegar a suplir nuevas necesidades del sistema, por lo que los individuos pueden experimentar cambios que no siempre son percibidos directamente, según afirma Álvarez (2015).

Redes sociales. Laso (2006) define las redes sociales, desde la teoría matemática de redes y grupos, como la estructura de los vínculos capaces de unir y establecer estados de cercanía entre las personas o distintos sistemas a través de espacios virtuales y aplicaciones capaces de trasmitir un flujo de información. 
Existen características predominantes en la consolidación de los vínculos, comprensión que parte desde la postura sistémica en la que se postulan cinco ejes fundamentales: la conformación de la relación y vicisitudes que favorecen la emergencia del conflicto, los motivos para permanecer en la relación, las fuentes de tensión, el motivo de consulta y los efectos de la intervención, según Herrera, Torres y Hernández (2013). La comprensión de las creencias y los pensamientos que influyen en la construcción de los vínculos se basan en los principios del pensamiento complejo, desde lo que se plantea como la racionalidad y los métodos transdisciplinarios, ante una comprensión desde el enfoque sistémico, la complejidad, el constructivismo y el paradigma ecológico que parten del mundo subjetivo y lo que integra al individuo. Los vínculos son lo que conectan a las personas sin medir el tiempo y la distancia, capaces de atribuir y conservar solidas las relaciones que se establecen desde la simbolización que se genera; los vínculos se construyen a través de la consolidación del lenguaje, como lo mencionan Hernández y Bravo (2004).

Aunado a ello, el lenguaje es capaz de promover entonces las simbolizaciones afectivas que fortalecen los lazos; para esto nombran dos tipos de vínculos. Los vínculos reales son aquellos que se dan o se cristalizan por los medios de comunicación en cuanto al aspecto afectivo, estos vínculos son capaces de demostrar representaciones del afecto que surgen en las parejas como entes naturales en la relación. Por su parte, los vínculos virtuales surgen a través de imaginarios, potenciales e ideales de las parejas, según lo expuesto por Gabelli (2014). Es así que los usos tecnológicos amplían las zonas de observación y vigilancia del otro, replanteando las fronteras entre la autonomía y el control y reforzando componentes del modelo del amor romántico, la presencia en la distancia y la exigencia de inmediatez, así como el stalking (sobreinformación, espionaje y acoso) (Rodríguez y Rodríguez, 2016).

Redunda en lo anterior la investigación llevada a cabo por Casero y Algaba (2016) en el archipiélago de las Islas Baleares, en España, titulada "Relación de pareja, uso de redes sociales y mensajería instantánea en los estudiantes de la Universidad de las Islas Baleares". Esta tiene como objetivo describir el patrón de uso de dispositivos interactivos y su nexo con la situación de relación de pareja; para ello fue aplicado un cuestionario a 776 estudiantes por medio de un muestreo mixto y polietápico mediante pruebas de independencia, comparaciones de medias y análisis de la varianza. Fueron tomados los parámetros definidos para ello, como por ejemplo el uso de las redes sociales y datos sociodemográficos académicos. Se analizaron múltiples variables como género, edad, si tenian pareja, tiempo de relación, carrera, curso actual y resultados académicos, entre otros. Como resultado se logra identificar que Facebook y Whatsapp son las redes con más uso, hasta el punto de servir para pasar el aburrimiento en el salón de clases.

Asimismo, Toledo y Molina (2014) publican su investigación en la Facultad de Filosofía de la Universidad del Azuay, denominada "Las redes sociales y su influencia en el comportamiento de los adolescentes". Este estudio fue realizado en cuatro colegios de la cuidad de Cuenca en Ecuador, con la finalidad de analizar la influencia de las redes sociales tomando las variables social, personal, familiar y académica. Para este fin, se construyó un cuestionario para los estudiantes y profesores en el cual participaron 444 personas. 
Los estudiantes afirmaron que para comunicarse usan teléfono, tablet y computadora, y utilizan las redes sociales Facebook, Twitter, Youtube y Whatsapp, hasta el punto de que llegan a ser complementarias en la vida cotidiana, por lo que los estudiantes pasan gran tiempo inmersos en ellas. A su vez, reportan que la finalidad del uso de estas redes se basa en buscar información académica, para conocer amigos, por curiosidad y por último para socializar e interactuar entre la red de amigos (Andrade, Aguilar, Sorto y Benegas, 2015).

Romo (2008) llevó a cabo en México la investigación denominada "Estudiantes universitarios y sus relaciones de pareja de sus experiencias y proyectos de vida". Esta investigación pretende aportar al estudio de alumnos jóvenes, debido a la necesidad de evidenciar los cambios en los diferentes escenarios de los estudiantes y sus perspectivas. Para esto se realizaron entrevistas similares a las de tipo autobiográficas y relatos de vida a nueve mujeres y siete hombres con una edad de veinte años aproximadamente. Lograron evidenciar que la mayor parte de los entrevistados han establecido alguna relación de pareja, las cuales van desde relaciones tranquilas hasta relaciones desgastantes basadas en celos.

Por su parte, García y Enciso (2014) en Tepic, Nayarit (México) realizan una investigación denominada "Influencia del Facebook en la relación de pareja", la cual buscó conocer cómo Facebook llega a afectar la relación de pareja en estudiantes y personal administrativo en la Universidad Autónoma de Nayarit, por lo que se buscaba identificar la frecuencia de permanencia en esta red social, el tipo de información que se compartían y el nivel de molestia que generaba esta tanto a hombres como a mujeres. Para esto se aplicó una encuesta que contiene once ítems, a treinta personas; se seleccionaron quince hombres y quince mujeres con una edad de veinte a cuarenta años que usan Facebook y que al momento de aplicar sostienen una relación de pareja. Por medio de esta se puede evidenciar que $40 \%$ de los encuestados mencionaron disgusto con su pareja por algunos los comentarios o likes ${ }^{1}$ que se publican en su cuenta. Se trata desde luego de mencionar que las redes sociales son un elemento positivo para las relaciones de pareja; aunque se pierda el lenguaje no verbal, estas redes permiten el uso de emoticones los cuales logran reemplazar este lenguaje, lo que permite facilitar la comunicación y mantener una relación (Reyes y Ramírez, 2015).

Por otro lado, Rajeev y Jobilal (2017) plantean en la Universidad de Amrita Kollam, en India, una investigación denominada "Effects of Social Media on Social Relationships: A Descriptive Study on the Impact of Mobile Phones among Youth Population", en la que mencionan los efectos de los medios de comunicación social en las relaciones sociales y el impacto de los teléfonos móviles entre la población juvenil. Se encontró que los usuarios son afectados masivamente por estas redes de comunicación, teniendo en cuenta que son utilizadas para comunicarse con amigos, familiares y demás, así como para generar trabajos, de manera que ofrecen una pequeña biografía de la vida de cada sujeto que active su red social, por lo que existen diversas maneras de estar inmersos en ella.

La información recolectada tiene que ver con que las personas se han incorporado a las redes sociales a tal punto que estas llegan a tener millones de usuarios. Esto sirve para

1 Término del idioma inglés que hace referencia a la acción en las redes sociales para dar a conocer el gusto de un usuario por una publicación. 
facilitar un apoyo consistente entre la práctica y el interés que brindan las herramientas de información y comunicación; así lo afirman Boyd y Ellison (2007), con respecto a una investigación de sitios de redes sociales definición, historia y becas titulada "Social Network Sities: Definition, History, and Scholarship" Ilevada a cabo por Smith (2010). Así es que las redes sociales han impactado la vida de las personas hasta el punto de disminuir las relaciones naturales que se presentan, haciendo que las personas se centren en su vida como usuarios a partir de la conectividad que ofrecen estas redes, lo que ocasiona que las personas estén en sí mismas aisladas del mundo real, según lo afirman Hampton, Sessions, Rainie y Purcell (2011).

Por otro lado, dentro de la investigación realizada por Bailey (2009), denominada "Couple relationships: Communication and conflict resolution. CFLE, family and human development", se encontró que los hombres y las mujeres utilizan de igual manera las redes sociales, pero sus fines difieren. En el caso del género masculino, las redes son utilizadas para cuestiones de la vida laboral, asuntos deportivos y la resolución de conflictos; por su parte, el género femenino se focaliza en su emocionalidad y la comunicación que pueden brindar con personas cercanas a estas.

Debido a lo anteriormente planteado, cabe mencionar que las redes sociales aportan una oportunidad para interactuar y demostrar una imagen de sí mismo, de manera que la persona se expresa y se integra con otros, y se da a conocer mediante pequeñas características en cada perfil; de esta manera, las redes son utiliadas como un medio de interacción, así que es más fácil establecer relaciones cercanas que redundan en el plano sentimental, por lo que también pueden originar problemas en la dinámica relacional debido al mal uso de estas, como lo afirman López, López y Galán (s.f.).

Estas situaciones son tomadas como un tema de alto interés para la sociedad, por lo que dentro de la investigación se pretende brindar aportes sobre la información de los elementos o herramientas de las redes sociales, que pueden fortalecer investigaciones futuras dentro de la disciplina, que puedan dirigir acciones que fortalezcan el desarrollo terapéutico, razón por la cual en la presente investigación se buscó conocer el impacto en las relaciones afectivas al indagar la información de veinte parejas del departamento del Meta (Colombia) que utilizaran las redes sociales como medio de comunicación en sus dinámicas relacionales. Estas dinámicas llegan a influir en la construcción de los vínculos afectivos que se gestan en el interior de estas, lo cual es de vital importancia para los autores al abordar esta temática, ya que, si bien se trata de adoptar una posición ontológica frente al fenómeno observado, los autores también hacen parte del fenómeno al encontrarse dentro de circunstancias similares, sin llegar a desconocerlas.

Estas situaciones surgen a raíz del uso de las redes sociales y son representadas por las parejas, razón por la cual este tema es concebido como alto interés e impacto para la sociedad; es por esto que dentro de la investigación se pretende brindar aportes sobre la información de los elementos o herramientas de las redes sociales, que puedan fortalecer investigaciones futuras estén dirigidas al desarrollo terapéutico, dentro de la disciplina.

Como estrategia de investigación fueron utilizadas las verbalizaciones de las parejas participantes, los cual sirvió de insumo para lograr identifcar las categorias emergentes a través de entrevistas que fueron diseñadas mediante la configuración de un guión y que posteriormente se analizaron para obtener los resultados de estas. 


\section{Metodología}

La investigación se fundamentó en una metodología cualitativa, la cual busca comprender las experiencias y emociones de los participantes a través de los significados que se dan de la percepción del mundo al que se vincula el individuo y su manera de relacionarse e interactuar a través de la interpretación de un fenómeno dado, lo que lleva a producir datos descriptivos desde las palabras propias de las personas (Quevedo y Castaño, 2002). Por lo tanto, se ahonda en los fenómenos desde la perspectiva de los participantes, en relación con su entorno y tomando en cuenta su discurso en la construcción de las interpretaciones, de manera que se explora y describe para así generar perspectivas teóricas, lo que implica que sea más flexible, y permite hacer una recolección y análisis de datos desde los fenómenos culturales o sociales en vez de limitarse a dar un resultado numérico (Hernández, Fernández, y Baptista, 2010).

Esta investigación toma como método lo fenomenológico (Montero, 2007) que busca interpretar el fenómeno desde lo cotidiano y la representación subjetiva de cada individuo; este parte de la observación el análisis y la reflexión que se da a través de la percepción del mundo en torno al individuo como sentimientos, sueños, deseos, imaginaciones, sensaciones, recuerdos, etc. (Vargas, 2010).

Para la recolección de la información se utilizó la entrevista semiestructurada, la cual fue desarrollada bajo parametros de validez de contenido a partir de la estructuración de sus apartados, los cuales fueron calificados por expertos, mediante la incorporación dentro de sus ítems los constructos necesarios para lograr la intrepretación de las experiencias de los participantes a través de guiones que orientaron las preguntas en pro de la interpretacion de las narrativas.

Finalmente, se tiene en cuenta el enfoque sistémico dentro de la estructura metodológica en la forma como la producción de los vínculos que generan las redes sociales afecta de una forma u otra la relación, por lo que se hace un análisis de estas reacciones a través de las verbalizaciones de los participantes.

Características de los participantes. La información fue recolectada de una muestra poblacional de veinte parejas heterosexuales que tuvieran las características que los investigadores delimitaron, tales como el género, masculino y femenino, y que las edades oscilaran entre los quince y los veintidós años de edad.

La población se eligió a partir de un caso-tipo, con el fin de obtener información relevante y concisa del fenómeno observado. Se busca tener en cuenta cualidades y características importantes que surgen dentro de la propia realidad, como valores, atributos, creencias y aquellos significados que nacen de la perspectiva del entorno, la muestra de casos tipo, además, resulta ser adecuada desde el estudio con perspectiva fenomenológica propuesto por Hernández, Fernández y Baptista(2010).

Procedimientos de muestreo. Dentro de la investigación cualitativa existen varias técnicas que permiten acceder a la información, dependiendo de la población, por lo que como herramienta para recolectar la información se utilizó la entrevista semiestructurada, al ser esta una técnica frecuentemente utilizada para este tipo de diseños (Cerda, 1991). 
Es por ello que a través de este instrumento se logra la construcción conjunta de nuevos significados en los temas expuestos por los entrevistadores, con un carácter conversacional que emerge para conocer los significados que le dan los entrevistados al mundo, a partir de sus propias experiencias, de manera que se crea un clima de comunicación que facilita la transmisión de la información (Ozona y Pérez, 2004).

Por último, los resultados fueron tomados desde la categoría "vínculos", en la cual se realizó una matríz de datos dentro de la cual emergieron cuatro subcategorías: "estabilidad", "reciprocidad", "comunicación" y "fuentes de tensión". Estas fueron tomadas a fin de ahondar en los relatos de los actores y dar el orden respectivo a la información recolectada; así se ubicaron las respuestas de los participantes desde las preguntas planteadas.

\section{Resultados}

Funamentado el estudio en los procesos de vinculación se logra evidenciar en las narrativas de las parejas participantes subcategorías tales como "vínculos reales", "vínculos virtuales", "comunicación", "estabilidad", "reciprocidad" y "fuentes de tensión" (Hernández y Bravo, 2010).

Categoría vínculos. En esta categoría se ubica la unión de las personas, dentro de la cual se establece una conexión temporo-espacial a través de simbolizaciones que fortalecen el mantenimiento de la relación, incluso ante la distancia. En este sentido las relaciones de pareja adoptan aspectos importantes que retroalimentan los momentos vividos al igual que los vínculos, lo cual genera cambios progresivos en el sistema relacional, así como limites internos de la pareja, de manera que se originan cambios afectivos en el funcionamiento de esta (Hernández y Bravo, 2004).

Por tal motivo, emergen vínculos reales y virtuales, de manera que se suplen necesidades físicas o virtuales ante una constante interacción emocional a través de la comprensión que se obtuvo de los relatos de los actores, quienes dieron cuenta de sus actitudes emocionales respecto a sus expresiones dentro de las redes sociales, lo que desgolsa subcategorías que demuestran la interpretación de dichas verbalizaciones.

Subcategoría vínculo real. Estos vínculos se pueden evidenciar por medio de distintas representaciones y afectos que dan cuenta de la esencia de la relación. A su vez, los vínculos pueden interpretarse de dos formas diferentes, pues se componen al mismo tiempo de violencia y pacificación, alineación y autonomía (Hernández, Fernández y Baptista, 2010). Las parejas refirieron sus relaciones como "normales", tal que tienen rutinas y salen a divertirse juntos. Por su parte, refirieron tener el constructo "tiempo" como una constante inmersa en la relación sentimental, por lo que los participantes señalaron que entre más tiempo tuviera la relación, mayor sería el vínculo formado.

Subcategoría vinculo virtual. Se refiere a la conexión que se da entre las personas por medio de imaginarios y representaciones que se dan a través de la interacción (Hernández, Fernández y Baptista, 2010), por lo que en este estudio las parejas refieren tener mayor seguridad al publicar sus estados afectivos en las redes sociales, 
publicar fotos y escribirse palabras afectuosas que les generan algún tipo de tranquilidad y les ubica dentro de un relación seria. Además, esto les genera una apropiación y sensación de importancia del vínculo virtual, ya que las redes sociales hacen parte de sus expresiones afectuosas del concepto "amor" que sienten y comparten en su vida diaria, de manera que esta se convierte en una necesidad mutua que da funcionamiento al sistema relacional.

Subcategoría comunicación. Se basa en la interacción que existe entre los individuos desde las acciones que realizan y el valor de la transmisión de un mensaje capaz de otorgar un significado, tanto de forma verbal como no verbal (Feixas, Muñoz, Compañ y Montesano, 2016), por lo que los sujetos participantes emplean un medio de comunicación desde redes sociales que proporciona en las parejas estados de cercanía, bienestar mutuo e incluso desconfianza por un malestar emocional ante los celos, en cuyo caso son las mujeres quienes más frecuentan estas redes (Gómez, 2014).

Generalmente las parejas tienen la necesidad de expresar el afecto que sienten por el otro, en este sentido las redes proporcionan herramientas al dar la posibilidad de mostrar reacciones emocionales a través de símbolos (me gusta, me encanta, me divierte, me enoja o me asombra), que son conocidos como "estados emocionales" y que son idóneos para que los usuarios tengan la capacidad de representar su emocionalidad en sus escirtos.

Teniendo en cuenta lo anterior, se evidencia que para las parejas, publicar un estado "me gusta" respesentado con el color azul les parece algo normal, pero que al momento de observar que su pareja publica un estado con el simbolo "me encanta" representado con un corazón de color rojo es mucho más comprometedor, debido a que este tiene la capacidad de hacer sentir incómoda a la pareja y se acrecienta al momento de que se presente con mayor frecuencia hacia otra persona (García, 2013).

Subcategoría estabilidad. Es importante anotar en esta subcategoría, que el equilibrio del sistema se da a través de la homeostasis con el fin de adaptarse a las diferentes transformaciones del desarrollo, no solo individual sino en la pareja vista como un sistema (Espinal, Gimeno y González, s.f).

Teniendo en cuenta lo anotado anteriormente, se evidencia que la estabilidad es mediada por el tiempo de duración del sistema relacional de la pareja y puede ser un elemento cambiante, en el que la pareja tiene la capacidad de adaptarse a dichos cambios, lo que genera en sus miembros la homeostasis, ya que a través del uso de las redes sociales otorgaron la oportunidad de conocerse, relacionarse y permanecer en constante comunicación de forma asincrona por motivos de distancia (Gómez y Marín, 2017).

Subcategoría reciprocidad. Identificado como el intercambio del comportamiento mutuo entre la pareja, en el que se da la armonía por medio de los espacios de comunicación a través de una forma equitativa (Espinal, Gimeno y González, s.f.), se anota que en la relación se presenta un comportamiento mutuo de armonía a través de los afectos y representaciones que se dan en el fortalecimiento de la relación por medio de las redes sociales, por lo que dan cuenta de que al tener control de las contraseñas de seguridad de sus parejas, las posiciona en un lugar de estabilidad y confianza. 
Los participantes afirman que las redes no son para producir problemas; mencionan que, al subir algún mensaje a las redes, este debe ser visto por sus parejas y etiquetado², pues si no hay este tipo de reciprocidad en la información, se generan problemas.

Subcategoría fuentes de tensión. Son las situaciones estresantes que surgen en los individuos intervinientes en el sistema capaces de generar cambios en el ciclo vital y ocasionar relaciones conflictivas (Herrera, Torres y Hernández, 2013).

Las redes sociales generan inconvenientes en las parejas, lo que ocasiona una ruptura en la estabilidad del sistema, como es el caso de la comunicación sincrónica, entendida a esta como la acción de estar en un intercambio directo de mensajes. Dicha acción es capaz de crear una inestabilidad emocional (Gay, s.f.). Al estar en constante interacción y suplir sus necesidades mutuas, estas parejas llegan a incorporar las redes en su vida, al punto que llegan a hacerlas partes del sistema, así como de su forma de actuar, conocer y distinguir todas las acciones que se generan alrededor de estas, de manera que toman relevancia las simbolizaciones que ofrecen la redes y generan en algunas ocasiones agrado y desagrado dependiendo a quien vayan dirigidas las reacciones, por lo que al momento de generar nuevas conexiones con otros sistemas puede evidenciarse como un hecho amenazante que desestabiliza el sistema relacional de la pareja, lo cual puede llevar a rupturas a nivel emocional. Así pues, las parejas vivenciaron esta situación de manera que al no tener control de las redes sociales de su pareja, se generaba angustia por el hecho de que posiblemente estaban escondiendo información que le comprometiera en la estabilidad, ya que sentían un sinsabor respectoa a lo que estaba haciendo su pareja, lo cual llevaba a un sentimiento de desasosiego.

\section{Conclusiones}

Al obtener diferentes posturas frente a la percepción que se tiene con respecto a las redes sociales y la manera en que influyen dentro de la dinámica relacional, desde la comprensión de la categoría de vínculo y las subcategorías que de esta emergen, se rescatan los aportes de la postura sistémica desde las narrativas de las parejas participantes, a partir de las experiencias propias y su vinculación en redes sociales como WhatsApp, Facebook e Instagram. Estas vivencias son tomadas como empíricas; a su vez, se toman los postulados teóricos que dan las bases para la comprensión de los resultados a la luz de la postura sistémica (Hoffman, 1992).

Cabe anotar que las redes sociales cambian la forma de actuar y de comportarse de los usuarios, al regular la manera de involucrarse como pareja. Así pues, se organiza y retroalimenta su funcionalidad al tener claras las limitaciones que se plantean, no solo en la manera física o presencial entre la pareja sino en cuanto a las reglas o el compromiso que se adquiere al momento de tener esta vida virtual. Garibay (2013) plantea que es importante tener parámetros en la relación para su formalización y proyecciones conjuntas, por lo que las formas de vinculación de forma real y virtual no son iguales, puesto que existen los vínculos que se dan al momento. Allí se toma lo real; es decir, las representaciones de acciones y sentimientos inmediatas como el compartir, salir, hablar de frente, etc. (Hernández, Fernández y Baptista, 2010).

2 Término que se refiere a la acción por medio de la cual una persona es mencionada en una publicación en las redes sociales, lo cual denota interés porque esta se entere de la información. 
Por otro lado, la emocionalidad que se presenta de manera virtual es capaz de perdurar en el tiempo, no solo creando sentimientos y reacciones inmediatas al momento de recibir la información, de leerla o verla, sino que también puede llegar a ser generadora de distintas emociones ya que se puede volver a recordar y leer en cualquier momento; en este sentido, las redes sociales pueden retener sentimientos, recuerdos e incluso llegar a fortalecer el vínculo al momento de estar quebrantado, al punto de reemplazarlo por una experiencia previa positiva ya que se conoce el fenómeno que se presenta, por lo que todo esto se da como un medio de homeostasis debido a que la pareja se adapta al cambio con las redes sociales desde una forma de recurso (Espinal, Gimeno y González, s.f).

De igual forma se menciona que es impredecible que la pareja reconozca el cambio que se presenta en el sistema, ya que en distintas ocasiones el cambio no es observable y apenas se puede percibir a la luz del investigador, como se resalta en la cibernética de segundo orden desde Brunett y Morell (2001), "el observador está inmerso en el fenómeno que observa", de tal manera que las parejas experimentan cambios que se pueden producir por la integración entre su estado y una amenaza interna en su propio entorno, como lo señalan Perinat, Lalueza y Sadurnì (2003) respecto a que se dan mecanismos de autorregulación del sistema ante el cambio que se presenta, conocido como "recurso", por lo que se debe recibir la información del medio dentro del sistema relacional, es decir que, mediante las herramientas que brindan, las redes sociales ofrecen oportunidades, elementos o aspectos que llegar a suplir las necesidades que para la pareja llegan a ser importantes o esenciales en la emocionalidad (Morín, 1997).

Frente a lo anteriormente planteado, se retoma la morfogénesis; como lo afirma Garibay (2013) "es la tendencia al crecimiento, además que cada sistema cuenta con diferentes niveles de organización" (p. 7), ya que el sistema relacional está en constante cambio y por instinto se dan nuevas tendencias al crecimiento mutuo. De esta manera, el sistema relacional de la pareja funciona de forma conjunta en relación a la interacción que se presenta con las redes sociales, a tal punto de caracterizar el sistema desde la sinergia, ya que se presenta una acción recíproca que se crea en la vinculación de la pareja. En este sentido corrobora que la sinergia estudia el funcionamiento que se da en el sistema de forma conjunta, capaz de conducir a la funcionalidad y regir los procesos organizativos que se presentan en la estructura del sistema (Los Santos, Xòchitl y Godoy, 2009).

Por su parte, Sánchez y Medina (2013) afirman que la estabilidad se da a partir del tiempo; esto se plantea en las parejas que hicieron parte de la investigación, dentro de la subcategoría "estabilidad", la cual se evidencia a través del tiempo de duración de la relación, las dinámicas compartidas, las pautas establecidas en la interacción con el contexto y como pareja. Los actores sostienen una relación consensuada de más de dos años, que se consolida a través de experiencias y dificultades que transforman el sistema relacional al cual hacen parte. Dicha modificación se presenta en la homeostasis dado que esta es la forma como la pareja se adapta al cambio, lo cual se configura en la interacción entre la pareja y la relación con la interacción en redes sociales, lo que produce un significado diferente en las dinámicas relacionales (Espinal, Gimeno y González, s.f.). 
Por otro lado, Feixas, Muñoz, Compañ y Montesano (2016) definen la comunicación como un "mecanismo de interacción necesario entre dos o más individuos" que resalta la idea de que es imposible no comunicarse desde la teoría de la comunicación humana. Así, en el primer axioma se propone que el individuo emite conductas a través del lenguaje no verbal y genera una comunicación, tal que produce un método de decodificación a la información que se pretende transmitir. En el caso que se toma para el estudio, este tipo de conductas se materializan a través de los símbolos que usan en la descomposición de un mensaje las redes sociales, las cuales proporcionan diferentes representaciones conocidas como "emoticones", que simbolozan las emociones mediante diferentes figuras, además de permitir interactuar con pares y sistemas familiares e intercambiar información personal con los demás, de tal manera que se crea una necesidad de constante interacción capaz de fortalecer y mantener el afecto y transmitir un estado de cercanía (Garibay, 2013).

Las parejas fortalecen sus relaciones a través de momentos significativos, al compartir a través del lenguaje y la comunicación, los cuales son capaces de generar vínculos reales que unen y mantienen sólida la relación. Estos momentos también se pueden trasmitir por el uso de las redes sociales que se dan por medio de una conexión temporo-espacial que genera vínculos virtuales idóneos. Esta conexión se puede reflejar en las parejas ya que aunque mantienen un nivel de distancia por los sitios donde se encuentran, las redes sociales les proporcionan un servicio importante en la consolidación de los vínculos, los cuales los hacen permanecer en constante interacción de cercanía y permiten la creación de imaginarios por medio de una comunicación simbólica (Hernández y Bravo, 2004).

Ahora bien, en algunos casos de parejas posesivas, cada miembro del sistema tiene acceso a la privacidad de las redes sociales del otro, pues de otra manera se crearían posturas de desconfianza que quebrantarían las conexiones de armonía. Por otra parte, el no tener acceso a la privacidad de la pareja indica asumir un grado de confianza para el mantenimiento de esta armonía, ya que las parejas establecen ciertos límites con otros nodos que anteriormente hayan establecido una conexión cercana, y evitan dar reacciones de "me encanta" a publicaciones, para exigir un grado de respeto al momento de sostener una conversación, con el fin de evitar tensiones y posibilitar así el funcionamiento de la relación. Esta es una práctica más propicia a la tranquilidad emocional de la pareja ya que al generar este tipo de tensiones se evitan disgustos (Espinal, Gimeno y González, s.f.).

Al mismo tiempo existen elementos estresantes que influyen de manera negativa en la relación de pareja por medio de los símbolos, que resultan ser situaciones estresantes para las parejas, como por ejemplo los comentarios (según el género de quien trasmite el mensaje), las reacciones entre "me gusta" o "me encanta", entendido este como el generador de más tensión en el funcionamiento del sistema. Como lo refieren las parejas participantes, es "molesto" ver siempre una misma persona que le dé "me gusta" y aun peor un "me encanta" a la pareja; dichos símbolos brindan un grado de importancia dependiendo de qué tan repetitivo es el mensaje que se trasmitió. De igual forma ocurre con las funcionalidades de las redes sociales como la conexión sincrónica que brinda la aplicación de WhatsApp; en este caso específicamente al "estar en línea" (que respresenta que la persona esta viendo su entorno virtual y se encuentra en disposición para comunicarse) y no hacer un intercambio directo del mensaje ocasiona conflictos capaces de generar cambios momentáneos en la dinámica relacional o hasta relaciones 
conflictivas (Herrera, Torres y Hernández, 2013).

Conviene entonces detenerse a ver que las parejas en su interacción con el contexto se vinculan no solo de manera presencial sino virtual como una forma circular a través de lo que hace parte del sistema, interactuando con otros medios, encontrando participación de otros subsistemas que suplen el funcionamiento de este, de manera que las herramientas de las redes sociales se vuelven generadoras de otras emociones que llegan a suplir o sustituir sentimientos negativos por positivos (Satir, 2002).

Análogamente cabe mencionar que los sistemas relacionales que se presentan dentro de esta investigación acogen y permiten que las redes sociales regulen el comportamiento, sentimientos y pensamientos, que no son percibidos directamente en las parejas, a tal punto que se generan cambios y pautas que dirigen la adaptación de la relación al medio vinculado (Marcos, 2009).

De las circunstancias mencionadas, nace el hecho de que las redes sociales transfieren mensajes por medio de los distintos canales de comunicación mediante símbolos que pueden generar sentimientos, teniendo en cuenta los tipos de comunicación sincrónica y asincrónica, entendidas como formas directas o indirectas de comunicar un mensaje, por lo que se resalta que el vínculo se da por medio de esta comunicación que ofrecen las herramientas de las aplicaciones como Facebook, WhatsApp e Instagram. Esto hace que el vínculo se presente también desde un medio virtual y sea capaz de ser trasmitido y transformado en un comportamiento emitido que se llega a reflejar; es decir que la comunicación está presente siempre, bien sea de manera virtual o real, ya que no es posible no comunicarse (Toledo y Molina, 2014).

De la misma manera se pueden llegar a dar cambios de forma positiva o negativa, puesto que el vínculo puede llegar a ser fortalecido por este medio de las redes sociales, o puede también llegar a ser desafiado o amenazado, desde la amplitud que existe en la conexión con otros subsistemas ante los cambios que se identifican en la dinámica relacional de la pareja, por lo que se logra observar en la investigación que los vínculos reales pueden pasar a un segundo plano, al ser más importantes para la pareja los "vínculos virtuales", debido a la importancia que se les da a las redes sociales en la acción conjunta de la relación (Maureira, 2011) de manera que las redes sociales regulan los comportamientos de manera virtual, generando comportamientos y significados en lo real, pero las parejas no tienen conciencia de las implicaciones del uso de las redes en su relación (Malagón, Cuestas y Reyes, 2014).

Es intersante observar el fenomeno desde el factor tiempo, ya que la comunicación virtual favorece el vínculo en tanto es perdurable en los textos, y estos pueden ser retomados posteriormente por los integrantes, lo que genera dificultades en el vínculo, lo cual se puede evidenciar en los símbolos como fuentes estresantes en la relación, como es el caso de la famosa acción de "dejar en visto", que no es más que observar el acuse del recibido por parte del otro y no producir ninguna reacción al respecto (Lozares, 2016).

Se concluye entonces que la interacción a partir de las redes sociales muestra un influjo importante en el desarrollo de las mismas y que las emocionalidaes tienen un papel protagónico en el devenir de los comportamientos de los actores frente al manejo de redes que inciden en las dinámicas relacones de las parejas, quienes permiten ser permeadeas 
por la órbita de la comunicación en la medida que estas afectan la interacción entre los sujetos y sus dinámicas; por lo que el aporte al proceso de investigación se sustenta en que el tema de las redes sociales involucra a todas las personas de diferentes edades, estrato socioeconómico y género, de manera que da una amplia información de cómo esto afecta de una u otra manera positivamente, en el fortalecimiento del vínculo a través de la preservación de los límites de la relación, desde elementos temporo-espaciales que ofrecen las redes sociales al suplir estados de cercanía; o por otro lado negativamente, ante los celos, la desconfianza en termino de compartirse la contraseña para estar vigilados, etc. Se llega así a abordar esta problemática formada por las redes sociales en las relaciones de pareja, desde la comprensión de cómo las parejas son conscientes de las transformaciones a través de las redes sociales.

Además, se logra evidenciar que en la literatura poco se encuentra con respecto a abordajes que vinculen las redes sociales y las relaciones de pareja, al ser este tema novedoso y pertinente para la época. Es por esto que aquí se aportan alternativas referentes a problemáticas de los sistemas relacionales de la pareja, en la que se presenten rupturas o modificaciones en el proceso vincular, y en la que se incorporan los sentimientos y las emociones de la misma desde las herramientas que ofrecen las redes sociales.

\section{Referencias}

Álvarez, E.L. (2015). Modelo psicodinámico y modelo sistémico en trabajo social. Ecuador: Universidad de Cuenca. Recuperado de: http://dspace.ucuenca.edu.ec/ bitstream/123456789/21533/1/tesis.pdf

Andrade, F.R.; Aguilar, M.C.; Sorto, L.M. y Benegas, Y.T. (2015). Ventajas y desventajas de las redes sociales en las relaciones de pareja. Universidad pedagógica nacional Francisco Morazán. Recuperado de: https://metodologiadeinvestigacioncuantitativa. files.wordpress.com/2015/05/ventajas-y-desventajas-de-las-redes-sociales-enrelaciones-de-pareja.pdf

Bailey, S. (2009). Couple Relationships: Communication and Conflict Resolution. CFLE, Family and Human Development Specialist. Recuperado de: https://www. msuextension.org/health/documents/MT200917HR.pdf

Boyd, D. y Ellison, N. (2007). Social Network Sities: Definition, History, and Scholarship. Michigan State University. Recuperado de: http://mimosa.pntic.mec.es/mvera1/ textos/redessociales.pdf

Brunett, I. y Morell, A. (2001). Epistemología y cibernética. Recuperado de: https://ddd. uab.cat/pub/papers/02102862n65/02102862n65p31.pdf

Casero, A. y Algaba, I. (2016). Relación de pareja, uso de redes sociales y mensajería instantánea en los estudiantes de la Universidad de las Islas Baleares. Revista de la facultad de ciencias sociales universidad nacional. Universidad de las Islas Baleares España, 36(53). Recuperado de: http://www.revistas.una.ac.cr/index.php/abra/ article/view/8771/pdf 
Castellanos, C. (2015). Influencia de las redes sociales en la relación de pareja de estudiantesdelaUniversidadRafael Landívar. Asunción, Guatemala: UniversidadRafael Landívar. Recuperado de: http://recursosbiblio.url.edu.gt/tesiseortiz/2015/05/42/ Castellanos-Camila.pdf

Cerda, H. (1991). Elementos de la investigación. [Tesis de maestría]. Universidad Nacional Abierta y a Distancia (UNAD). Bogotá, Colombia. Recuperado de: http:// postgrado.una.edu.ve/metodologia2/paginas/cerda7.pdf

Cornejo, M. y Tapia, M.L. (2011). Es nombrada redes sociales y relaciones interpersonales en internet social. Universidad Nacional de San Luis, Argentina. Recuperado de: http://fundamentos.unsl.edu.ar/pdf/articulo-24-219.pdf

Cuervo, R. (2013). Parejas viables que perduran en el tiempo. Diversitas. Perspectivas en Psicología, 9(2). Universidad Santo Tomas. Recuperado de: http://www.redalyc. org/articulo.oa?id $=67932397002$

Espinal, I.; Gimeno, A. y González, F. (s.f). El enfoque sistémico en los estudios sobre familia. Universidad Autónoma de Santo Domingo (UASD) y Centro Cultural Poveda. Santo Domingo, República Dominicana. Recuperado de: http://www.uv.es/jugar2/ Enfoque\%20Sistemico.pdf

Feixas, G.; Muñoz, D;, Compañ, V. y Montesano, A. (2016). El Modelo Sistémico en la Intervención Familiar. Universidad de Barcelona. Recuperado de: http://diposit. ub.edu/dspace/bitstream/2445/31584/6/Modelo Sistemico Enero2016.pdf

Gabelli, F.M. (2014). Teoría de Selección Sexual y Origen de los Sexos. Universidad de Buenos Aires. Recuperado de: http://www.psi.uba.ar/academica/carrerasdegrado/ psicologia/sitios catedras/electivas/090 comportamiento/material/teoria de seleccion sexual.pdf

Gainza, C._(2003). Sociedad, Estado y tecnología: ¿qué pasa hoy con nuestras sociedades? Revista de sociología. Universidad de Chile. Recuperado de: http:// www.facso.uchile.cl/publicaciones/sociologia/articulos/17/1707-Gainza.pdf

García, A. (2013). Una lectura del amor desde la sociología: algunas dimensiones de análisis social. Revista Scielo. Recuperado de: http://www.scielo.org.mx/pdf/soc/ v28n80/v28n80a5.pdf

García, L. y Enciso, R. (2014). Influencia del Facebook en la relación de pareja. Revista Edúcateconciencia. 4(4). Nayarit, México. Recuperado de: http://tecnocientifica. com.mx/volumenes/V4A17.pdf

Garibay, S. (2013). Enfoque sistémico: Una introducción a la psicoterapia familiar. México: Editorial El Manual Moderno.

Gay, A. (s.f). Enfoque sistémico. Recuperado de: http://www.manuelugarte.org/ modulos/biblioteca/g/texto 2 aquiles gay.pdf 
Balaguera-Rojas et al - El vínculo relacional de pareja y las redes sociales: una mirada desde la cotidianidad

Gómez, M. (2014). Relaciones románticas constructivas "RRC" Evaluación de un Programa Psicoeducativo para el Desarrollo de Relaciones de Pareja Constructivas en la Adolescencia. Bogotá: Universidad de los Andes. Recuperado de: https:// documentodegrado.uniandes.edu.co/documentos/200913413 fecha 20140124 hora 190156 parte $1 . p d f$

Gómez, K.K., y Marín, J.S. (2017). Impacto que genera las redes sociales en la conducta del adolescente y en sus relaciones interpersonales en Iberoamérica los ultimo diez años. Universidad Cooperativa de Colombia. Recuperado de: http://repository.ucc. edu.co/bitstream/ucc/747/1/Impacto $\% 20$ que $\% 20$ generan $\% 20$ las $\% 20$ redes $\% 20$ sociales $\% 20$ en $\% 201$ a $\% 20$ conducta $\% 20$ del $\% 20$ adolescente $\% 20 y \% 20$ en $\% 20$ sus $\% 20$ relaciones $\% 20$ interpersonales $\% 20$ en $\% 20$ Iberoam $\%$ C $3 \%$ A 9 rica $\% 20$ los\%20\%C3\%BAltimos\%2010\%20a\%C3\%B10s.pdf

Hampton, K. ; Sessions, L. ; Rainie, L. y Purcell, K. (2011). Social networking sites and our lives How people's trust, personal relationships, and civic and political involvement are connected to their use of social networking sites and other technologies. Pew Research Center's Internet y American Life Project. Washington, D.C.: University of Pennsylvania. Recuperado de: http://www.pewinternet.org/files/old-media/Files/ Reports/2011/PIP\%20-\%20Social\%20networking\%20sites\%20and\%20our\%20 lives.pdf

Hernández, A. (2010). Vínculos, individuación y ecología humana: hacia una psicología clínica compleja. Bogotá, Colombia.

Hernández, A. y Bravo, F. (2004). Vínculos, redes y ecología. Hallazgos revista de investigaciones. Universidad Santo Tomas. Recuperado de: http://revistas.usta. edu.co/index.php/hallazgos/article/viewFile/1575/1736

Hernández, R.; Fernández, C. y Baptista, P. (2010). Metodología de la investigación. $5^{a}$ ed. México: MC Graw Hill. Recuperado de: https://www.esup.edu.pe/descargas/ dep investigacion/Metodologia\%20de\%20la\%20investigaci\%C3\%B3n\%205ta\%20 Edici\%C3\%B3n.pdf

Herrera, C.; Torres, J.A. y Hernández, A. (2013). Comprensión de las dinámicas de relación de parejas del mismo sexo a partir de la intervención clínica sistémica. Bogotá: Pontifica Universidad Javeriana, International Academy of LGTB Psychology and Related Fileds. Recuperado de: https://www.liberarte.co/docs/Comprensionde-dinamicas-relacion-parejas-mismo-sexo.pdf

Hoffman, L. (1992). Fundamentos de la terapia familiar: Un marco conceptual para el cambio de sistemas. 2a ed. México: Fondo de cultura económica. Recuperado de: http://www.verticespsicologos.com/sites/default/files/Fundamentos-de-la-terapiafamiliar.pdf

Laso, E. (2006). Análisis de redes sociales y terapia familiar sistémica. Psicología en positivo: psicoterapia por internet. Recuperado de: http://estebanlaso.com/?p=381 
Los Santos, A.; Xòchitl, M. y Godoy, D. (2009). Web 3.0: integración de la web semántica y la web 2.0. Recuperado de: http://www.albertolsa.com/wp-content/ uploads/2009/07/redessociales-web-30-integracion-de-la-web-semantica-y-laweb-20-Ios-santos-nava-godoy.pdf

López, M.; López, V. y Galán, E. (s.f. ). Redes sociales de internet y adolescente. psicología del desarrollo y educación. Recuperado de: http://www.websatafi.com/joomla/ attachments/125 REDES\%20SOCIALES\%20PUBLICAR.pdf

Lozares, C. (1996). Teoría de las redes sociales. Universidad autónoma de Barcelona. Departamento de Sociología. Recuperado de: http://www.raco.cat/index.php/ papers/article/viewFile/25386/58613

Malagón, L.; Cuestas, J. y Reyes, I. (2014). Influencia del Facebook en el mantenimiento de las relaciones de pareja en estudiantes de la Universidad Piloto de Colombia. Bogotá. Recuperado de: http://polux.unipiloto.edu.co:8080/00002118.pdf

Marcos, M. (2009). Evolución de los servicios de redes sociales en internet. Revista el profesional de la información 18(5). Recuperado de: http://www. elprofesionaldelainformacion.com/contenidos/2009/septiembre/09.pdf

Maureira, F.C. (2011). Los cuatro componentes de la relación de pareja. Universidad Santiago de Chile. Recuperado de: http://www.iztacala.unam.mx/carreras/ psicologia/psiclin/vol14num1/Vol14No1Art18.pdf

Ministerio de Tecnologías de la Información y las Comunicaciones. (2015). Colombia lidera en internet libre y rápido en América. Bogotá: Gobierno de Colombia. Recuperado de: http://www.mintic.gov.co/portal/604/w3-article-11345.html

Montero, J. (2007). La fenomenología de la conciencia en e. Husserl. Universitas Philosophica. Univercidad javeriana. Bogotá. Recuperado de: http://revistas. javeriana.edu.co/index.php/vniphilosophica/article/viewFile/11271/9185

Morín, E. (1997). Introducción al Pensamiento Complejo. Recuperado de: http:// cursoenlineasincostoedgarmorin.org/images/descargables/Morin Introduccion al pensamiento complejo.pdf

Ozona, L. y Pérez, A. (2004). La entrevista semiestructurada. Notas sobre una práctica metodológica desde una perspectiva de género. Universidad Nacional del Comahue. Recuperado de: http://www.biblioteca.unlpam.edu.ar/pubpdf/aljaba/ n09a19ozonas.pdf

Perinat, A.; Lalueza, J. y Sadurnì, M. (2003). Psicología del desarrollo: un enfoque sistémico una nueva edición revisada y ampliada. Barcelona: Editorial UOC.

Quevedo, R. y Castaño, C. (2002). Introducción a la metodología de investigación cualitativa. Revista de Psicodidactica. Universidad del País Vasco, 14. Recuperado de: http://www.redalyc.org/pdf/175/17501402.pdf 
Balaguera-Rojas et al - El vínculo relacional de pareja y las redes sociales: una mirada desde la cotidianidad

Reyes, B. y Ramírez, M. (2015). Calidaddela relación de pareja y comunicación vía redessociales. México: Universidad de Colima. Recuperado de: http://www.academia.edu/19973582/ Calidad de Relaci\%C3\%B3n de Pareja y Comunicaci\%C3\%B3n V\%C3\%ADa Redes Sociales

Rajeev, M. y Jobilal, M. (2017). Effects of Social Media on Social Relationships: A Descriptive Study on the Impact of Mobile Phones among Youth Population. International Research Journal of Social Sciences, 4(2). Recuperado de: http://www.isca.in/IJSS/Archive/v4/ i2/4.ISCA-IRJSS-2014-259.pdf

Ricoy, C. (2006). Contribución sobre los paradigmas de investigación. Revista do Centro de Educação, 31(1). Universidad Federal de Santa María, Brasil. Recuperado de: http:// www.redalyc.org/pdf/1171/117117257002.pdf

Rodríguez, T. y Rodríguez, Z. (2016). El amor y las nuevas tecnologías: experiencias de comunicación y conflicto. Revista Scielo. Universidad de Guadalajara, México. Recuperado de: http://www.scielo.org.mx/pdf/comso/n25/n25a2.pdf

Romo, J. (2008). Estudiantes universitarios y sus relaciones de pareja. De sus experiencias y proyectos de vida. Revista Mexicana de Investigación Educativa, 13(38). Recuperado de: http://www.redalyc.org/pdf/140/14003806.pdf

Sánchez, E. y Medina, A. (2013). Comunicación emocional y problemas conyugales en parejas que asisten a psicoterapia sistémica: una relación recursiva. Pontificia Universidad Javeriana. Bogotá, Colombia. Recuperado de: https://repository.javeriana.edu.co/ bitstream/handle/10554/3850/SanchezBedoyaEmma201; jsessionid=F0C57A3C132D3 00F7ABB0A42367507C9?sequence $=1$

Satir, V. (2002). Nuevas relaciones humanas en el núcleo familiar, 2a ed. México: Editorial Pax México, Librería Carlos Cesarman, S.A. Recuperado de: https:// lafamiliacomosistema2014. files.wordpress.com/2016/03/virginia-satir-relacioneshumanas-en-el-nucleo-familiar-1.pdf

Smith, D. (2010). Social Networks the Next Generation. Recuperado de: file:///C:/Users/ forero/Downloads/Social Networking - the next generation.pdf

Toledo, K. y Molina, G. (2014). Las redes sociales y su influencia en el comportamiento de los adolescentes. Universidad del Azuay facultad de filosofía de la cuidad de Cuenca, Ecuador. Recuperado de: http://dspace.uazuay.edu.ec/bitstream/datos/3659/1/10335.PDF

Urizar, M. (2012). Vínculo afectivo y sus trastornos. Galdakao-Bilbao. Recuperado de: http:// www.avpap.org/documentos/bilbao2012/DesarrolloAfectivoAVPap.pdf

Vallejos, O. (s.f). Introducción a internet. Recuperado de: http://ing.unne.edu.ar/pub/ internet.pdf

Vargas, X. (2010). ¿Cómo hacer investigación cualitativa? Unidad académica de contexto. ITESO. Recuperado de: https://es.scribd.com/doc/48098212/Como-hacer-investigacioncualitativa-LIBRO-DE-TEXTO 